\title{
Microtubule encounter-based catastrophe in Arabidopsis cortical microtubule arrays
}

\author{
Zhihai Chi and Chris Ambrose*
}

\begin{abstract}
Background: The cortical microtubules (CMTs) that line the plasma membrane of interphase plant cells are extensively studied owing to their importance in forming cell walls, and their usefulness as a model system for the study of MT dynamic instability and acentrosomal MT organization. CMTs influence the orientation and structure of cellulose microfibrils in the cell wall by cooperatively forming arrays of varied patterns from parallel to netted. These CMT patterns are controlled by the combined activities of MT dynamic instability and MT-MT interactions. However, it is an open question as to how CMT patterns may feedback to influence CMT dynamics and interactions.

Results: To address this question, we investigated the effects of CMT array patterning on encounter-based CMT catastrophe, which occurs when one CMT grows into another and is unable to cross over. We hypothesized that the varied CMT angles present in disordered (mixed CMTs) arrays will create more opportunities for MT-MT interactions, and thus increase encounter-based catastrophe rates and distribution. Using live-cell imaging of Arabidopsis cotyledon and leaf epidermal cells, we found that roughly $87 \%$ of catastrophes occur via the encounter-based mechanism, with the remainder occurring without encounter (free). When comparing ordered (parallel) and disordered (mixed orientation) CMT arrays, we found that disordered configurations show higher proportions of encounter-based catastrophe relative to free. Similarly, disordered CMT arrays have more catastrophes in general than ordered arrays. Encounter-based catastrophes were associated with frequent and sustained periods of pause prior to depolymerization, and CMTs with tight anchoring to the plasma membrane were more prone to undergo encounter-based catastrophe than weakly-attached ones. This suggests that encounter-based catastrophe has a mechanical basis, wherein MTs form physical barriers to one another. Lastly, we show that the commonly used measure of catastrophe frequencies $\left(F_{\text {cat }}\right)$ can also be influenced by CMT ordering and plasma membrane anchoring.

Conclusions: Our observations add a new layer of complexity to our current understanding of MT organization in plants, showing that not only do individual CMT dynamics influence CMT array organization, but that CMT organization itself has a strong effect on the behavior of individual MTs.
\end{abstract}

Keywords: Arabidopsis, Microtubule, Cytoskeleton, CLASP, Pavement epidermal cell, Microtubule-associated protein, clasp-1 mutant

\section{Background}

Microtubules (MTs) are polymers of $\alpha / \beta$-tubulin heterodimers that self-assemble into polar filaments with a fast-growing end, the plus end, and a relatively stable end, the minus end [1]. MTs play diverse roles at all stages of the eukaryotic cell cycle; guiding cell division, expansion and morphogenesis. To accomplish their varied tasks, MTs group together to form specialized arrays that are continuously remodeled by a process

\footnotetext{
* Correspondence: chris.ambrose@usask.ca

Department of Biology, University of Saskatchewan, 112 Science Place, Saskatoon, SK S7N 5E2, Canada

termed dynamic instability, wherein individual MT ends switch stochastically between growth and shrinkage through GTP hydrolysis [1,2]. Transitions from growth to shrinkage are termed catastrophe, and the transitions from shrinkage to growth are called rescue [3]. These parameters are biochemically modulated by MT-associated proteins (MAPs), which perform a variety of functions including stabilizing MTs through promoting polymerization of tubulin $[4,5]$, facilitating MT-MT interactions (e.g. crosslinking) [6, 7], and destabilizing MTs through increasing depolymerisation rate or severing MTs $[5,8]$. 
To orchestrate MT organization on a cellular scale, MT-organizing centers (MTOCs) are important structures that provide biochemical, mechanical, and positional information. By nucleating and tethering MTs, MTOCs create polarized groups of microtubules such as spindles and interphase cytoplasmic arrays. MTOCs are varied and ubiquitous throughout eukaryotes and include centrosomes in animal cells, spindle pole bodies in fungi [9], basal bodies in flagellated cells [9], the nuclear envelope in plants [10-17], and plastid in some algae [15]. However, in many cells, MTOCs are absent but MTs still obtain global ordering through self-organization, a process wherein global order emerges in a system from interactions between individual elements $[18,19]$. This "acentrosomal" MT organization is intensely studied in the interphase cortical array of plants, which consists of MTs that are laterally attached to the plasma membrane and undergo continuous dynamic behaviours. These Cortical MTs (CMTs) arrange in various orientations ranging from random/netted, parallel, and highly bundled. By guiding cellulose synthase complexes, CMT organization influences cell wall microfibril patterning, which in turn guides cell expansion patterns [20]. Since CMTs are restricted to the plane of the plasma membrane and highly dynamic, the probability of interactions between individual CMTs is much higher than MTs that are free to roam in three dimensions. Indeed numerous types of MT-MT interactions have been documented and shown to influence CMT arrangement $[19,21,22]$. These MT-MT interactions include bundle formation, collision-induced catastrophe [21], severing [23, 24], and nucleation of MTs from pre-existing MTs [25-27].

With respect to bundle formation and collision-induced catastrophe, when a growing CMT encounters another CMT, the relative angle between the two MTs determines whether the incoming MT will form a bundle with the barrier CMT or undergo catastrophe [21]. Specifically, when the contact angle between a growing plus end and the barrier MT is a small angle (for example, less than $\left.40^{\circ}\right)$, the plus ends reorients and continues growth alongside the barrier MT, forming a bundle. But when the incoming MT contacts the barrier MT more directly (i.e. at a large angle), the MT either depolymerizes (encounterbased catastrophe) or crosses over. From this, Dixit and Cyr developed a model for self-organization of CMTs into parallel arrays, wherein bundling speeds up parallel CMT formation, and encounter-based catastrophe favors elimination of CMTs with orientations not parallel to the predominant CMT orientation [21]. Using quantitative experimental data from CMT dynamics, numerous computer simulations and mathematical models have supported this model, but the relative contributions of bundling and encounter-based catastrophe are less clear [21, 28-36].
Whether CMT encounter-based catastrophe results from biochemical and/or mechanical mechanisms (or both) is not known. Several catastrophe-inducing MAPs have been found in plants, including kinesin-13A, MAP18 and ARK1kinesin [37-39]. However, since CMT catastrophe also occurs without MT-MT encounter, it is unclear as to whether these MAPs have any specificity/ preference for collision-induced catastrophe. In terms of mechanical forces, it is known that catastrophe can occur when a growing MT encounters a physical barrier. Specifically, the continued addition of tubulin subunits to the stalled MT plus end generates compressive force along the MT axis, which can induce MT bending and catastrophe [40-43]. In addition to MT-MT encounters, polymerization against cell edges can also induce CMT catastrophe $[44,45]$.

Given that MT-MT encounter outcomes are angledependent, it is possible that the highly variable arrangements of CMTs can influence rates of catastrophes, which may then feedback to CMT arrangements. In this study, we sought to investigate: (1) if CMT arrangement can influence catastrophe rates and spatial distribution; and (2) what the mechanistic underpinnings of encounterbased catastrophe are. To address these questions, we performed detailed quantifications of CMT arrangements, catastrophe types (encounter-based or free-based), spatial distributions, crossovers, and CMT-cortex attachment levels. We provide evidence for a strong contribution of force-induced catastrophe, and show that indeed CMT arrangement can influence catastrophe types and frequencies.

\section{Results}

Encounter-based catastrophes constitute the dominant source of CMT catastrophe

To visualize CMT dynamics, we performed confocal imaging using cotyledons and leaves of Arabidopsis seedlings constitutively expressing GFP fused to Arabidopsis TUBULIN BETA6 (GFP-TUB6) [46]. All data shown represent the combined data from leaves and cotyledons. The sharp cell edges present in meristematic and unexpanded cells are known to induce catastrophe when MTs grow into them [44], so in the current study we used mature epidermal cells in order to remove any potential cell edge effects on catastrophe behavior. The large size and rounded cell edges of these cells allowed sampling of large and relatively flat central areas of the outer cell face that were typically tens of microns away from nearby edges. For characterization of catastrophe types, we classified MT catastrophe into encounterbased catastrophe (Fig. 1a, c) or free catastrophe (Fig. 1b, d). Notably, we found that $87.6 \%$ of catastrophes in leaves and cotyledons were encounter-based, with the remaining $12.4 \%$ being not associated with encounter (Fig. 1e). The 


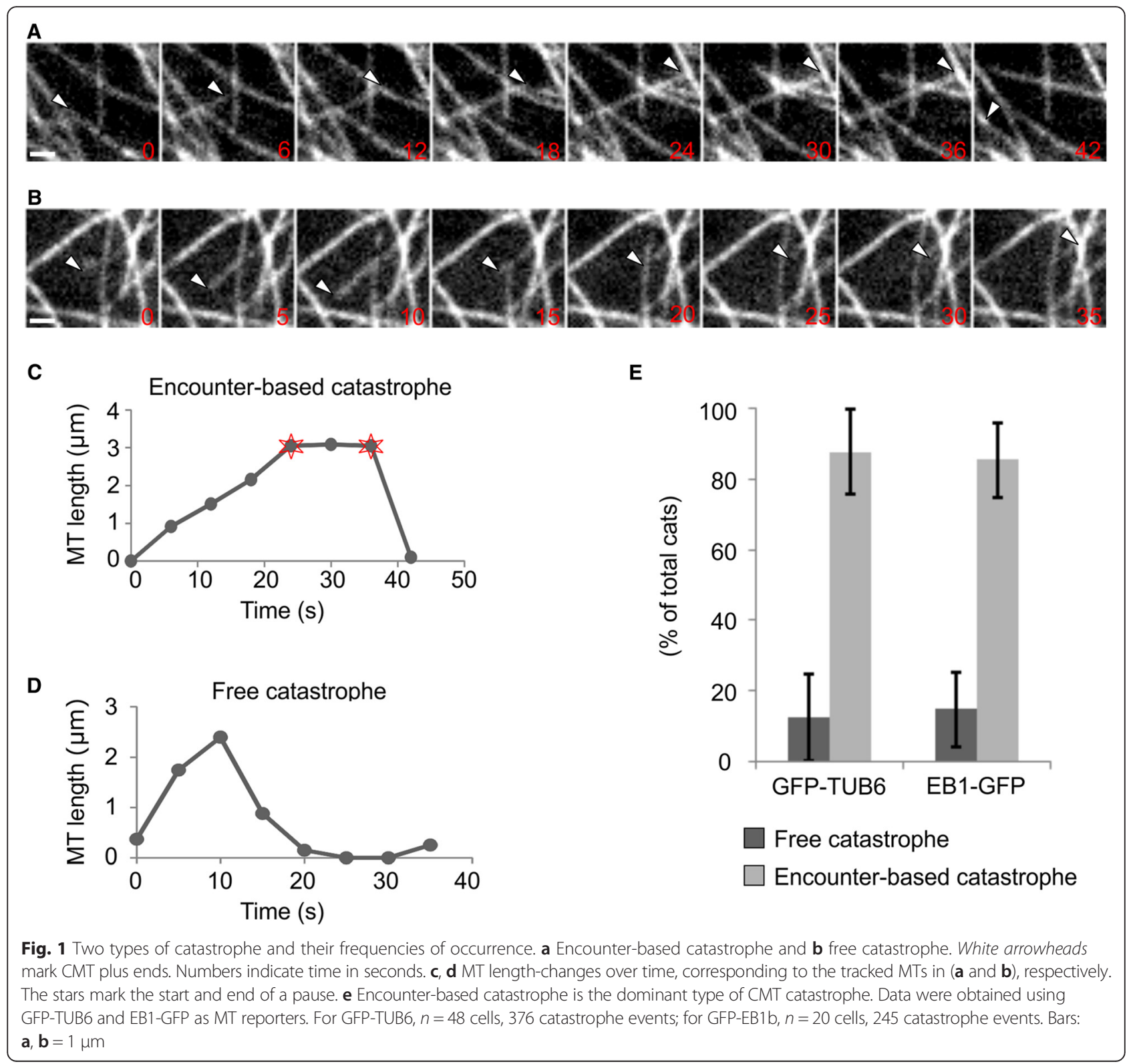

quantification of another line expressing 35S:GFP-EB1b showed a similar trend, with $85.3 \%$ of catastrophes being encounter-based (Fig. 1e).

\section{CMT organization influences the type and spatial} distribution of catastrophes

Based on these data, we hypothesized that compared to ordered (parallel) arrays, a highly disordered CMT array will provide more opportunities for CMT collision, and will thus have a larger proportion of encounter-based catastrophes compared to free catastrophes (i.e. a higher ratio of encounter-based to free). To test this, we measured CMT angles within parallel and disordered arrays (see Methods section for CMT angle measurements) and assessed the relative frequency of encounter- based catastrophes (i.e. \% encounter-based to total catastrophes). As a measure of array order, we used the standard deviation of CMTs angles. With this method, parallel arrays show smaller standard deviations in CMT angle than net-like arrays. This is illustrated in Fig. 2, which shows an example of a disordered array $(\mathrm{SD}=52)($ Fig. $2 \mathrm{a})$ and an example of an ordered array $(\mathrm{SD}=19)($ Fig. $2 \mathrm{~b})$. To ensure specificity of our data, SD and catastrophe ratios were measured on a cell-by-cell basis. We sampled 48 cells with varying degrees of order, and found a correlation between CMT array disorder and encounter-based catastrophes (Fig. 2c and d). Specifically, the more disordered a CMT array is, the higher the proportion of encounter-based catastrophes is. We observed a similar trend using 35S:GFP-EB1b lines (data not shown). These 

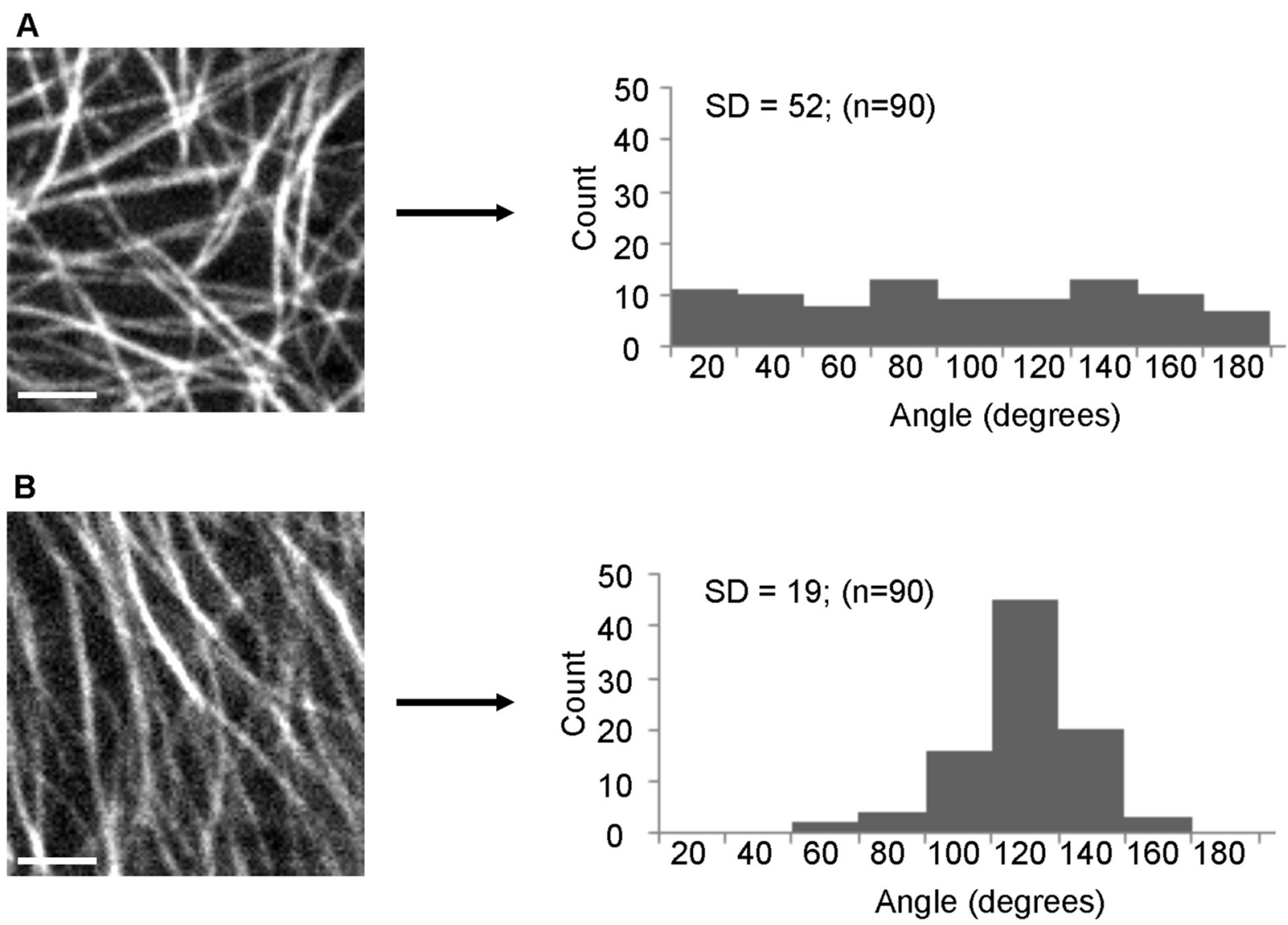

C

D
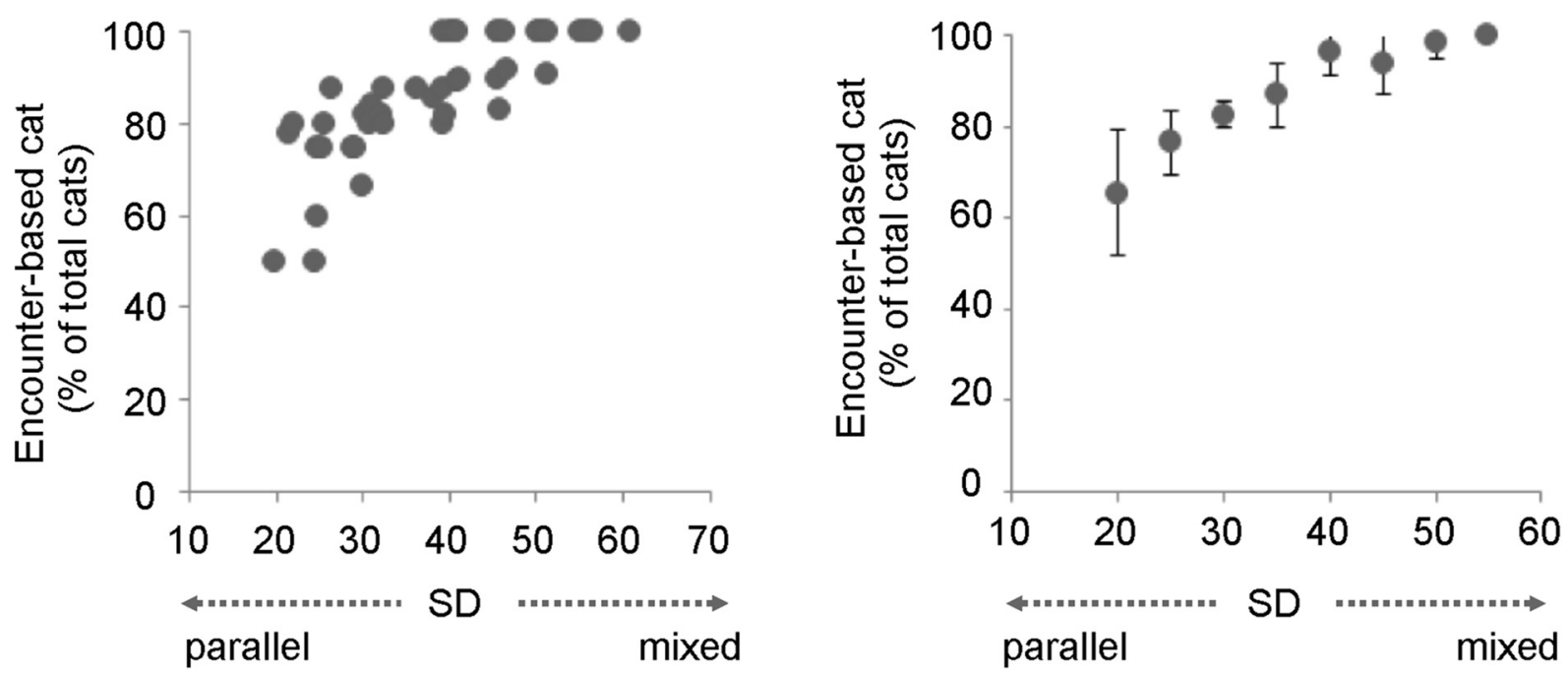

Fig. 2 The ratio of encounter-based to free catastrophe varies with CMT arrangement. Examples of disordered (a) and highly ordered CMTs (b). Histograms show CMT angle distributions corresponding to the images. c, d Disordered CMT arrays have a higher proportion of encounter-based catastrophes relative to free catastrophes. CMT order is expressed as the SD of CMT angles. $\mathbf{c}$ Un-binned data from individual cells (i.e. each point represents one cell); $\mathbf{d}$ data from (c) binned in five SD units. Bars: $\mathbf{a}, \mathbf{b}=2.5 \mu \mathrm{m}$ 
results indicate that CMT arrangement is an important determinant of catastrophe type.

Given this organizational influence on catastrophe type, we hypothesized that disordered CMT arrays will also have a higher number of catastrophe events in general (i.e. per unit area and time). We term this property as Density of Catastrophe $\left(D_{\text {cat }}\right)$ in order to clearly distinguish it from the dynamic instability parameter Catastrophe Frequency $\left(\mathrm{F}_{\mathrm{cat}}\right)$. Proper quantification of $D_{\text {cat }}$ presents a number of challenges. For example, simply measuring catastrophes per cellular area is problematic because the MT density itself within a given area would be a major determinant of density of catastrophe. Similarly, even normalizing for the area occupied by MTs is biased due to the fact that many MTs are very long, bundled, and stable, such that they span the entire region of observation and do not show observable MT end dynamics during the duration of the observation period. Based on these caveats, we chose to normalize density of catastrophe by expressing it as a function of the number of growing plus ends present within the observational region. To obtain simple and accurate counts of growing plus ends, we employed a subtractive technique similar to that used by Burnette et al. 2011 [47]. By subtracting pixel values between sequential time frames, only areas occupied by new MT growth are shown in the output image (Fig. 3a). Using this method, we indeed found a positive correlation between CMT disordering and density of catastrophe (Fig. 3b). We found a similar trend using GFP-EB1b lines as an indicator of plus end density (MT sidewall labeling was enough to detect the presence or absence of a barrier MT for each catastrophe) (Fig. 3c, d). These data indicate that CMT organization can influence the type and density of catastrophes.

\section{Strong MT-cortex attachment favors encounter-based catastrophe}

We next sought to investigate the mechanism governing encounter-based catastrophe. Given that MT polymerization against a physical barrier is known to induce MT bending and depolymerization $[44,48]$, and that the degree of CMT-cortex attachment influences the outcome of MT-MT interactions by imposing constraints on lateral motions of the MT polymer [30, 49], we hypothesized that when a weakly-attached CMT encounters a barrier MT, it will be more prone to lateral motions such as bending and detachment, which act to relieve the axial compression on the incoming MT, and hence reduce the probability of depolymerization. To test this, we quantified CMT behaviour in clasp-1 mutants, which show decreased CMT-cortex attachment [49]. To quantify the frequencies of encounter-based catastrophe, we classified encounter events as either cross-over or catastrophe (MT-MT bundling events were excluded). In general, cross-overs were far more

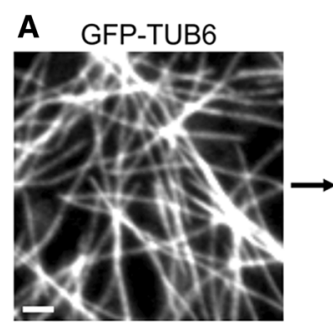

Original
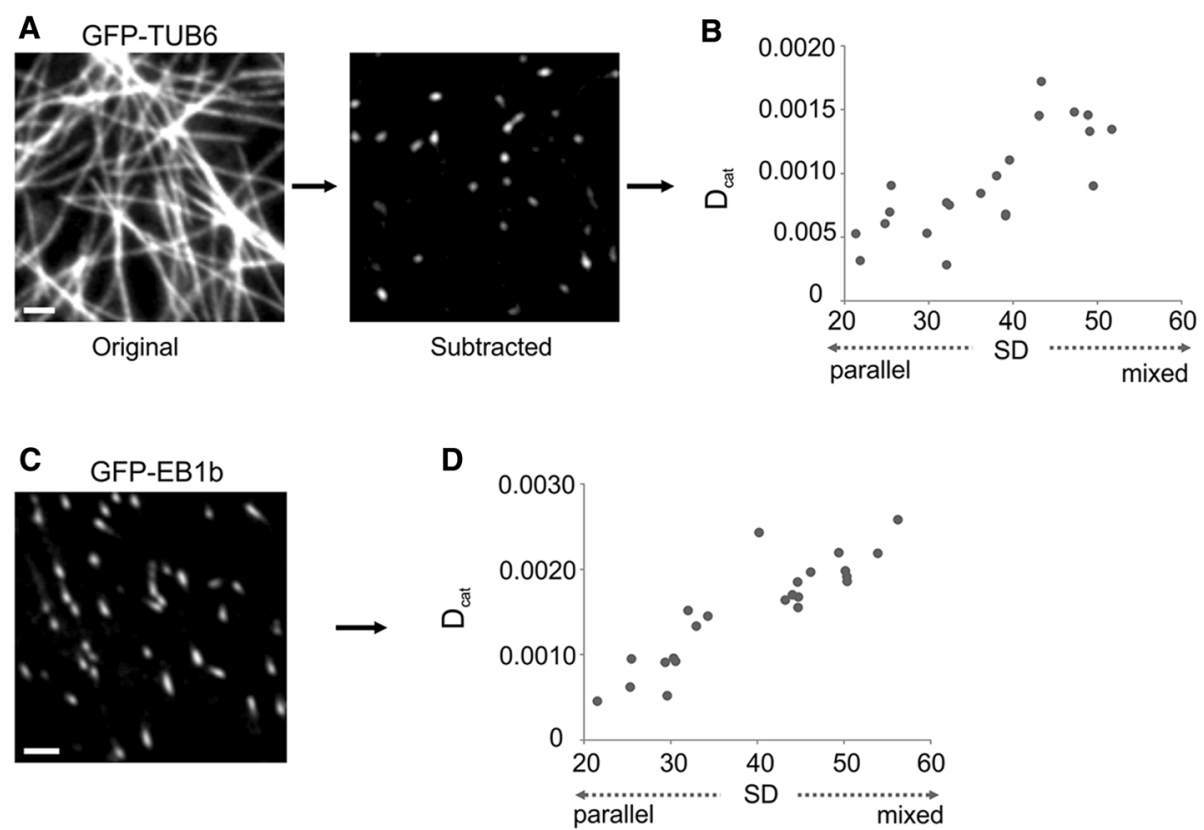

Fig. 3 Density of catastrophe $\left(D_{\text {cat }}\right)$ varies with CMT arrangement. a Example of subtracted image used for measurements to count growing plus ends with GFP-TUB6. b Density of catastrophe ( $D_{\text {cat }}$ increases with increasing CMT array disorder (expressed as SD of CMT angles). Measurements derived from GFP-TUB6 subtraction method. $\mathbf{c}$ Example of GFP-EB1b image used to count growing plus ends. $\mathbf{d}$ GFP-EB1b data shows a similar trend to GFP-TUB6 in (b). Bars: $\mathbf{a}, \mathbf{c}=2 \mu \mathrm{m}$ 
numerous than catastrophes in both genotypes, in agreement with previous findings in Arabidopsis [23]. Figure 4a shows two examples of MT crossovers, one of which grows in a straight line and eventually undergoes pause and encounter-based catastrophe (red), and another which shows partial detachment and swinging of the growing end as it grows across other MTs (green). This MT appears to be less anchored to the cortex because after it re-attaches, it encounters another MT and stalls, during which time it bends (presumable due to force of continued polymerization at the stalled plus end). Figure 4b shows a kymograph of an individual MT growing across several other MTs (which appear as vertical lines in the kymograph).

For quantification of crossover and catastrophes, measurements were normalized using the ratio of encounter- based catastrophe to total encounter events (i.e. catastrophe + cross-over). We found that CMTs in clasp-1 are more prone to cross over barrier MTs than wild type, and are less likely to undergo catastrophe (Fig. 4c). These data support the idea that strongly-attached MTs are prone to catastrophe because they are restricted to any lateral movement (which would dissipate axial compression), and are unable to detach and cross over the barrier MT.

\section{Encounter-based catastrophes exhibit a pause in growth before catastrophe}

During our analysis of catastrophe events, we noticed that the growth of an incoming CMT often pauses upon encounter with a barrier MT and is followed by catastrophe and depolymerization. Figure 5 a shows kymographs of free catastrophes without pause (left panel)
A

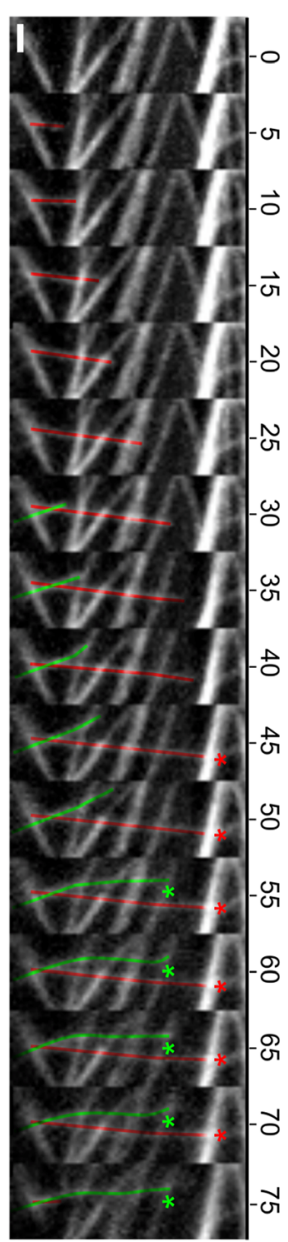

C

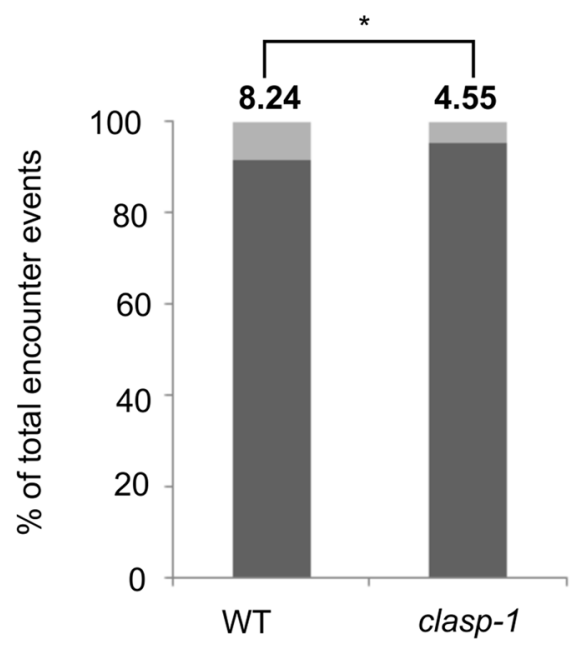

Catastrophe Crossover

B

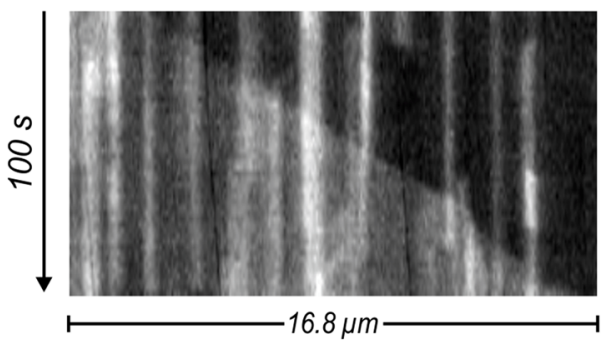

Fig. 4 Encounter-based catastrophe is related to CMT-cortex attachment. a Example of cross-over and encounter-based catastrophe in WT. Red line marks several cross-over events before pause and encounter-based catastrophe. Green line marks a detachment event as described in Results. Numbers indicate time in seconds. Both asterisks indicate pause events. Bars: $\mathbf{a}=2 \mu \mathrm{m}$. b Kymographs showing CMT with several cross-over events. c Quantification of crossover and encounter-based catastrophe. Asterisk indicates statistically significant difference of catastrophe frequency between WT and clasp-1 when $P<0.05 . n=10$ cells, 3675 encounter events 
A

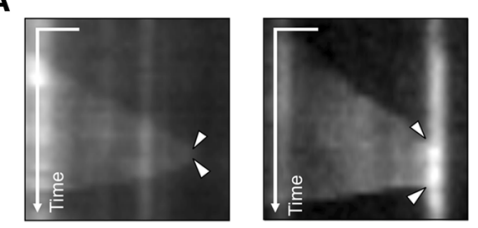

B



C

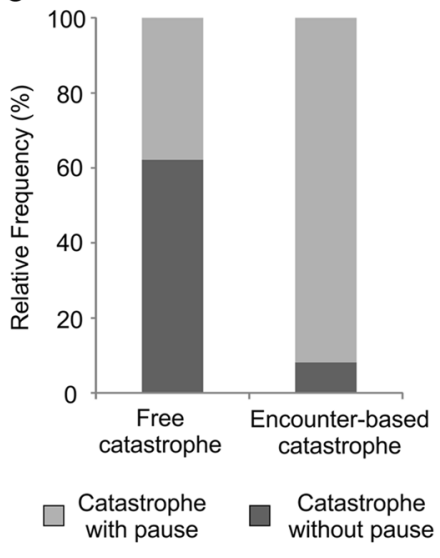

Fig. 5 CMTs with encounter-based catastrophe pause upon encounter with the barrier MT. a Kymographs showing free catastrophe without pause (left panel) and encounter-based catastrophe with pause (right panel). The arrowheads mark the start and the end of contact. Bars: The kymographs display a period of $76 \mathrm{~s}$, scale bar $=1 \mu \mathrm{m}$. $\mathbf{b}$ Histogram showing the distribution of pausing times for MTs that undergo encounterbased catastrophe. The pausing time ranges from 10 to $80 \mathrm{~s}$, and average time is $19.23 \mathrm{~s}$. c Encounter-based catastrophes are preceded by pause more frequently than are free catastrophes. $n=107$ cells, 119 free catastrophe and 1196 encounter-based catastrophe events

and encounter-based catastrophe with pause (right panel). As shown in the histogram in Fig. 5b, the time spent in pause state for encounter-based catastrophe event varies considerably, with a mean of $19 \pm 11 \mathrm{~s}$, and a maximum of $80 \mathrm{~s}$. For subsequent analysis, we defined pause as a state during which no observable MT growth is detectable for more than 5 s. Quantification of pause events revealed that $91.7 \%$ of encounter-based catastrophe events were preceded by pause (Fig. 5c). In contrast, only $37.8 \%$ of free catastrophes were preceded by pause, and the mean time for pause is $12 \pm 6 \mathrm{~s}(\sim 34 \%$ less than for encounterbased pauses) (Fig. 5c). Taken together, these data show that encounter of a growing CMT plus-end with a barrier MT greatly enhances the likelihood and duration of pause prior to depolymerization.

\section{CMT instability dynamics for catastrophe frequency vary with array organization}

Having found that CMT-cortex attachment and CMT organization both have effects on CMT catastrophes, we hypothesized that these factors may lead to inconsistencies in standard measurements of catastrophe frequencies $\left(\mathrm{F}_{\text {cat }}\right)$. To test this, we measured and compared $\mathrm{F}_{\text {cat }}$ values between disordered and parallel arrays; as well as between weakly-attached and strongly-attached CMT arrays (i.e. clasp-1 vs WT) (Results summarized in Table 1). $F_{\text {cat }}$ was calculated using the total number of catastrophe events divided by the total time spent in growth and pause, and only CMTs that show catastrophe at any point during its observation were used for measurements $[21,50,51]$. Notably, we found that CMTs showing free-type catastrophes have a significantly higher $F_{\text {cat }}(0.030 \pm 0.009$ events/s $)$ compared to those having encounter-based catastrophes $(0.021 \pm 0.002$ events/s) (Table 1). However, this difference is heavily biased from the longer durations of pause associated with encounter-based catastrophes (i.e. longer pauses increase total measured MT growth times, and thus decreased $\mathrm{F}_{\text {cat }}$ ). Despite these differences, when comparing $\mathrm{F}_{\text {cat }}$ between disordered CMT arrays (defined as arrays with SDs between 50 and 60) and well-ordered (defined as arrays with SDs between 20 and 30), we found no significant difference in $F_{\text {cat }}$ values (disordered $=0.022 \pm 0.002$ events $/ \mathrm{s}$; Ordered $=0.021 \pm 0.002$ events $/ \mathrm{s}$ ) (Table 1). This suggests that although free catastrophes do have higher average $F_{\text {cat }}$ values than encounter-based catastrophe, they are not numerous enough to significantly influence overall cellular $\mathrm{F}_{\text {cat }}$ measurements. However, when assessing the influence of CMT-cortex attachment on $\mathrm{F}_{\text {cat }}$, we found that wildtype cells have a significantly higher $F_{\text {cat }}(0.021 \pm 0.001$ events/s) than clasp-1 $(0.014 \pm 0.001$ events/s) (Table 1$)$.

Table 1 Catastrophe frequency varies between different catastrophe types and cell types. Values are means \pm s.d. For catastrophe frequencies, $n=12$ cells and 191 catastrophe events; for $F_{\text {cat }}$ in both WT and clasp, $n=10$ cells and 234 catastrophe events

\begin{tabular}{ll}
\hline CMT catastrophe frequencies (events/s) & \\
\hline General catastrophe $F_{\text {cat }}$ & $0.022 \pm 0.002$ \\
Free catastrophe $F_{\text {cat }}$ & $0.030 \pm 0.009$ \\
Encounter-based catastrophe $F_{\text {cat }}$ & $0.021 \pm 0.002$ \\
Less organized CMT catastrophe $F_{\text {cat }}$ & $0.021 \pm 0.003$ \\
Well organized CMT catastrophe $F_{\text {cat }}$ & $0.022 \pm 0.002$ \\
General catastrophe in WT $F_{\text {cat }}$ & $0.021 \pm 0.001$ \\
General catastrophe in clasp $F_{\text {cat }}$ & $0.014 \pm 0.001$ \\
\hline
\end{tabular}


This is consistent with the above observations of WT CMTs being less likely to cross over the barrier MT (Fig. 4c).

\section{Discussion}

Since the first characterization of plant CMT dynamic instability [2], several studies have made dynamic measurements using a variety of cell types, mutants, and drug treatments to investigate the mechanisms of CMT organization $[4,5,20,46,50-59]$. From these studies, several types of MT-MT interactions were discovered and have emerged to be major players in defining CMT organization and behaviour. Our findings build on this, showing that CMT organization can itself feedback to influence CMT interactions. The observation that 87\% of CMT catastrophes are associated with MT-MT encounter indicate that MT catastrophe in particular is heavily influenced by MT-MT interactions and CMT array organization. Observations by Zhang et al. (2013) and Wightman et al. (2007) that MTs frequently depolymerize following their katanin-dependent severing at MT-MT crossover points suggests that severing rates may also be influenced by overall CMT organization (since disorganized arrays have more MT-MT crossover points) [23, 60].

Based on our findings that MT organization can influence the catastrophe properties of individual MT is important to consider when assessing the in vivo functions of plant MAPs because it may confound assumed measurements of biochemical activities. This is illustrated in the current study by our clasp-1 mutant data. Without taking into account MT-MT influences on dynamic instability, our observations of reduced $\mathrm{F}_{\text {cat }}$ in clasp-1 mutants could be interpreted to indicate that CLASP functions biochemically to induce MT catastrophe, thereby acting as a MT de-stabilizer. However, numerous studies have shown that CLASP stabilizes MTs via promotion of pause and rescue in animals and fungi $[45,61,62]$. This apparent contradiction can be reconciled by our data, which suggest that the clasp-1 reduction in $\mathrm{F}_{\text {cat }}$ results at least in part from the enhanced MT cross-overs associated with weakly cortex-bound CMTs. CLASPs generally stabilize MTs specifically in localized regions of the cell such as cell edges $[29,44]$, chromosomal kinetochores [63], and between overlapping regions of interpolar spindle microtubules in fission yeast [64]. Therefore, measurements from different parts of a given cell can also yield seemingly contradictory data.

With respect to the mechanism of encounter-based catastrophe, our data suggest that in plants, mechanical forces on MTs play a major role in catastrophe induction. This applies not only to CMT encounter-based catastrophe, but also to cell edge-induced catastrophe [44]. Thus, when considering the cell as a whole, the bulk of CMT catastrophes occur either at cell edges [44] or via encounter-based catastrophe (current study). In both cases, catastrophe is preceded by behaviours characteristic of MT polymerization against a physical barrier such as prolonged pause and bending $[23,43,44,65,66]$.

\section{Conclusions}

Studies on CMT dynamics and organization in plants generally have focused on the mechanisms by which CMT behavior influences cellular CMT organization. The data presented in the current study show that the organization of CMTs within a cell also has strong influences on CMT dynamic behavior. Thus, a dynamic interplay exists between cellular CMT organization and individual CMT dynamics, indicating feedback between the two elements.

\section{Methods}

Plant materials and growth conditions

Transgenic Arabidopsis thaliana (Columbia-0) and clasp-1 lines expressing 35S:TUB6-GFP [49, 67], as well as WT plants expressing 35S:GFP-EB1b [68] were used for measurements. Prior to plating, all seeds were stored in the dark at $4{ }^{\circ} \mathrm{C}$ for $2 \mathrm{~d}$. Seeds were sterilized in $70 \%$ ethanol, rinsed twice with sterile water, and plated onto Petri dishes containing $1 / 2$ MS media, $1.0 \%$ agar, and $1 \%$ sucrose. Plates were wrapped with nescofilm (Azwell Inc.) and transferred to a $21{ }^{\circ} \mathrm{C}$ growth cabinet (with continuous light) and placed vertically. Young pavement cells of leaf and cotyledon cells were imaged at 3-4 days.

\section{Microscopy and image analysis}

All observations were performed on living cells. Cotyledons/leaves were cut at their base, mounted on to slides in Perfluoroperhydrophenanthrene (PP11) [69] under coverslip and sealed with wax. Images were obtained via point-scan confocal microscopy (Zeiss Meta 510 with Zeiss Axiovert 200M microscope, 63X water immersion), TIRF (Olympus IX83 with ANDOR iXon Ultra EMCCD camera, 100X oil immersion), and structured light illumination (Zeiss Apotome mounted on Zeiss Axio Imager Z1 with AxioCam MRm, 63x oil immersion). Time-lapse intervals ranged from 2-5 s. Images were processed with Image J software (http://rsb.info.nih.gov/ij/), and figures were assembled using Corel Draw software.

\section{CMT angle and density measurements}

For quantification of CMT array order, images were sampled by using five evenly-spaced horizontal rectangular boxes that were $2 \mu \mathrm{m}$ high and as wide as the image. The narrow height was used to remove any ambiguity of MT angle that may arise due to MTs that are bent to varying degrees. Only regions of cells that were covered by a sampling box were measured, which helped remove selection bias when using large cells. MT angles were defined relative to the horizontal axis of the image. 
Using a second method to measure CMT angles produced similar results. For this, a central area of the cell (away from edges) was sampled for up to 30 MTs.

For quantification of $\mathrm{SF}_{\text {cat }}$, subtractive analysis of sequential time frames was performed by duplicating the time-lapse image twice, and removing one to two frames from the start of the first duplicate, and removing the same number of frames from the end of the second duplicate. Using the image calculator function of ImageJ, the duplicates were then subtracted, and the resulting image was used to count growing plus ends. Since catastrophes were measured for the entire time series, we sampled plus end numbers at four evenly spaced time-points throughout the series and averaged the counts. 21 cells were sampled in total.

\section{Abbreviations \\ CLASP: clip-associated protein; CMT: cortical microtubule; $D_{\text {cat: }}$ Density of Catastrophe; EB1b: end-binding protein $1 \mathrm{~b}$; $\mathrm{F}_{\text {cat }}$ : frequency of catastrophe; GFP: green fluorescent protein; MAP: microtubule-associated protein; MT: microtubule.}

\section{Competing interests}

The authors declare that they have no competing interests.

\section{Authors' contributions}

ZC and CA performed experiments. ZC performed data analysis and figure assembly. ZC and CA wrote the manuscript. CA conceived the study. Both authors have read and approved the final version of the manuscript.

\section{Acknowledgements}

We would like to thank Dr. Eiko Kawamura for excellent technical assistance with TIRF microscopy experiments, and University of Saskatchewan Western College of Veterinary Medicine Microscopy Facility for use of the TIRF microscope. We thank the University of Saskatchewan Department of Biology Microscopy Facility for use of Zeiss microscopes.

This work was supported by the University Of Saskatchewan College Of Arts and Science Faculty and Recruitment Retention Plan's New Investigator Award, and by NSERC Discovery Grant 2015-05938.

Received: 15 October 2015 Accepted: 6 January 2016

Published online: 16 January 2016

\section{References}

1. Desai A, Mitchison TJ. Microtubule polymerization dynamics. Annu Rev Cell Dev Biol. 1997;13:83-117.

2. Shaw SL, Kamyar R, Ehrhardt DW. Sustained microtubule treadmilling in Arabidopsis cortical arrays. Science. 2003;300:1715-8.

3. Mitchison T, Kirschner M. Dynamic instability of microtubule growth. Nature. 1984:312:237-42

4. Yao M, Wakamatsu Y, Itoh TJ, Shoji T, Hashimoto T. Arabidopsis SPIRAL2 promotes uninterrupted microtubule growth by suppressing the pause state of microtubule dynamics. J Cell Sci. 2008;121:2372-81.

5. Kawamura E, Wasteneys GO. MOR1, the Arabidopsis thaliana homologue of Xenopus MAP215, promotes rapid growth and shrinkage, and suppresses the pausing of microtubules in vivo. J Cell Sci. 2008;121(Pt 24):4114-23.

6. Muller S, Smertenko A, Wagner V, Heinrich M, Hussey PJ, Hauser MT. The plant microtubule-associated protein AtMAP65-3/PLE is essential for cytokinetic phragmoplast function. Curr Biol. 2004;14:412-7.

7. Hussey PJ, Hawkins TJ, Igarashi H, Kaloriti D, Smertenko A. The plant cytoskeleton: recent advances in the study of the plant microtubuleassociated proteins MAP-65, MAP-190 and the Xenopus MAP215-like protein, MOR1. Plant Mol Biol. 2002:50:915-24.

8. Burk DH, Ye ZH. Alteration of oriented deposition of cellulose microfibrils by mutation of a katanin-like microtubule-severing protein. Plant Cell. 2002;14:2145-60.
9. Gundersen GG, Gomes ER, Wen Y. Cortical control of microtubule stability and polarization. Curr Opin Cell Biol. 2004;16:106-12.

10. Clayton L, Black CM, Lloyd CW. Microtubule nucleating sites in higher plant cells identified by an auto-antibody against pericentriolar material. J Cell Biol. 1985;101:319-24.

11. Wick S. The higher-plant mitotic apparatus - redistribution of microtubules, calmodulin and microtubule initiation material during its establishment. Cytobios. 1985:43:285-94.

12. Liu B, Marc J, Joshi HC, Palevitz BA. A gamma-tubulin-related protein associated with the microtubule arrays of higher plants in a cell cycledependent manner. J Cell Sci. 1993;104(Pt 4):1217-28.

13. Erhardt M, Stoppin-Mellet V, Campagne S, Canaday J, Mutterer J, Fabian T, et al. The plant Spc98p homologue colocalizes with gamma-tubulin at microtubule nucleation sites and is required for microtubule nucleation, J Cell Sci. 2002;115(Pt 11):2423-31.

14. Kumagai F, Nagata T, Yahara N, Moriyama Y, Horio T, Naoi K, et al. Gammatubulin distribution during cortical microtubule reorganization at the M/G1 interface in tobacco BY-2 cells. Eur J Cell Biol. 2003;82:43-51.

15. Brown RC, Lemmon BE. The pleiomorphic plant MTOC: An evolutionary perspective. J Integr Plant Biol. 2007:49:1142-53.

16. Seltzer V, Janski N, Canaday J, Herzog E, Erhardt M, Evrard J-L, et al. Arabidopsis GCP2 and GCP3 are part of a soluble $\gamma$-tubulin complex and have nuclear envelope targeting domains: Targeting of $\gamma$-tubulin complex proteins. Plant J. 2007:52:322-31.

17. Ambrose C, Wasteneys GO. Microtubule Initiation from the Nuclear Surface Controls Cortical Microtubule Growth Polarity and Orientation in Arabidopsis thaliana. Plant Cell Physiol. 2014;55:1636-45.

18. Hashimoto T, Kato T. Cortical control of plant microtubules. Curr Opin Plant Biol. 2006:9:5-11.

19. Ehrhardt DW, Shaw SL. Microtubule dynamics and organization in the plant cortical array. Annu Rev Plant Biol. 2006:57:859-75.

20. Dixit $R$, Cyr R. The cortical microtubule array: From dynamics to organization. Plant Cell. 2004;16:2546-52.

21. Dixit R, Cyr R. Encounters between dynamic cortical microtubules promote ordering of the cortical array through angle-dependent modifications of microtubule behavior. Plant Cell. 2004;16:3274-84.

22. Wasteneys GO, Ambrose JC. Spatial organization of plant cortical microtubules: close encounters of the 2D kind. Trends Cell Biol. 2009;19:62-71.

23. Wightman R, Turner SR. Severing at sites of microtubule crossover contributes to microtubule alignment in cortical arrays. Plant J. 2007:52:742-51

24. Lindeboom JJ, Nakamura M, Hibbel A, Shundyak K, Gutierrez R, Ketelaar T, et al. A mechanism for reorientation of cortical microtubule arrays driven by microtubule severing. Science. 2013;342:1245533.

25. Wasteneys GO, Jablonsky PP, Williamson RE. Assembly of purified brain tubulin at cortical and endoplasmic sites in perfused internodal cells of the alga Nitella tasmanica. Cell Biol Int Rep. 1989;13:513-28.

26. Murata T, Sonobe S, Baskin TI, Hyodo S, Hasezawa S, Nagata T, et al Microtubule-dependent microtubule nucleation based on recruitment of gamma-tubulin in higher plants. Nat Cell Biol. 2005;7:961-8.

27. Chan J, Sambade A, Calder G, Lloyd C. Arabidopsis cortical microtubules are initiated along, as well as branching from, existing microtubules. Plant Cell. 2009;21:2298-306

28. Allard JF, Wasteneys GO, Cytrynbaum EN. Mechanisms of Self-Organization of Cortical Microtubules in Plants Revealed by Computational Simulations. Mol Biol Cell. 2010;21:278-86.

29. Ambrose C, Wasteneys GO. Cell edges accumulate gamma tubulin complex components and nucleate microtubules following cytokinesis in Arabidopsis thaliana cells. PLoS One. 2011;6:e27423.

30. Baulin VA, Marques CM, Thalmann F. Collision induced spatial organization of microtubules. Biophys Chem. 2007;128:231-44.

31. Deinum EE, Tindemans SH, Mulder BM. Taking directions: the role of microtubule-bound nucleation in the self-organization of the plant cortical array. Phys Biol. 2011;8:056002.

32. Eren EC, Dixit R, Gautam N. A three-dimensional computer simulation model reveals the mechanisms for self-organization of plant cortical microtubules into oblique arrays. Mol Biol Cell. 2010;21:2674-84.

33. Eren EC, Gautam N, Dixit R. Computer simulation and mathematical models of the noncentrosomal plant cortical microtubule cytoskeleton. Cytoskelet Hoboken NJ. 2012;69:144-54. 
34. Hawkins RJ, Tindemans SH, Mulder BM. Model for the orientational ordering of the plant microtubule cortical array. Phys Rev E Stat Nonlin Soft Matter Phys. 2010;82(1 Pt 1):11911.

35. Shi X-Q, Ma Y-Q. Understanding phase behavior of plant cell cortex microtubule organization. Proc Natl Acad Sci. 2010;107:11709-14.

36. Tindemans SH, Hawkins RJ, Mulder BM. Survival of the aligned: ordering of the plant cortical microtubule array. Phys Rev Lett. 2010;104:58103.

37. Wang $X$, Zhu L, Liu B, Wang C, Jin L, Zhao Q, et al. Arabidopsis MICROTUBULE-ASSOCIATED PROTEIN18 Functions in Directional Cel Growth by Destabilizing Cortical Microtubules. Plant Cell Online. 2007;19:877-89

38. Oda Y, Fukuda H. Rho of Plant GTPase Signaling Regulates the Behavior of Arabidopsis Kinesin-13A to Establish Secondary Cell Wall Patterns. Plant Cell. 2013;25:4439-50.

39. Eng RC, Wasteneys GO. The microtubule plus-end tracking protein ARMADILLO-REPEAT KINESIN1 promotes microtubule catastrophe in Arabidopsis. Plant Cell. 2014;26:3372-86.

40. Tischer C, Brunner D, Dogterom M. Force- and kinesin-8-dependent effects in the spatial regulation of fission yeast microtubule dynamics. Mol Syst Biol. 2009;5:250.

41. Janson ME, Dogterom M. Scaling of microtubule force-velocity curves obtained at different tubulin concentrations. Phys Rev Lett. 2004;92:2723-36.

42. Dogterom M, Yurke B. Measurement of the Force-Velocity Relation for Growing Microtubules. Science. 1997;278:856-60.

43. Janson ME, de Dood ME, Dogterom M. Dynamic instability of microtubules is regulated by force. J Cell Biol. 2003;161:1029-34.

44. Ambrose C, Allard JF, Cytrynbaum EN, Wasteneys GO. A CLASP-modulated cell edge barrier mechanism drives cell-wide cortical microtubule organization in Arabidopsis. Nat Commun. 2011;2:430.

45. Mimori-Kiyosue Y, Grigoriev I, Lansbergen G, Sasaki H, Matsui C, Severin F, et al. CLASP1 and CLASP2 bind to EB1 and regulate microtubule plus-end dynamics at the cell cortex. J Cell Biol. 2005;168:141-53.

46. Nakamura M, Naoi K, Shoji T, Hashimoto T. Low concentrations of propyzamide and oryzalin alter microtubule dynamics in Arabidopsis epidermal cells. Plant Cell Physiol. 2004;45:1330-4.

47. Burnette DT, Sengupta P, Dai Y, Lippincott-Schwartz J, Kachar B. Bleaching/ blinking assisted localization microscopy for superresolution imaging using standard fluorescent molecules. Proc Natl Acad Sci. 2011;108:21081-6.

48. Laan L, Husson J, Munteanu EL, Kerssemakers JWJ, Dogterom M. Forcegeneration and dynamic instability of microtubule bundles. Proc Natl Acad Sci U S A. 2008;105:8920-5.

49. Ambrose JC, Wasteneys GO. CLASP modulates microtubule-cortex interaction during self-organization of acentrosomal microtubules. Mol Bio Cell. 2008;19:4730-7.

50. Dhonukshe P, Gadella TWJ. Alteration of microtubule dynamic instability during preprophase band formation revealed by yellow fluorescent proteinCLIP170 microtubule plus-end labeling. Plant Cell. 2003;15:597-611.

51. Vos JW, Dogterom M, Emons AMC. Microtubules become more dynamic but not shorter during preprophase band formation: A possible "searchand-capture" mechanism for microtubule translocation. Cell Motil Cytoskeleton. 2004;57:246-58.

52. Van Damme D, Bouget FY, Van Poucke K, Inze D, Geelen D. Molecular dissection of plant cytokinesis and phragmoplast structure: a survey of GFP-tagged proteins. Plant J. 2004;40:386-98.

53. DeBolt S, Gutierrez R, Ehrhardt DW, Melo CV, Ross L, Cutler SR, et al. Morlin, an inhibitor of cortical microtubule dynamics and cellulose synthase movement. Proc Natl Acad Sci U A. 2007;104:5854-9.

54. Galva C, Kirik V, Lindeboom JJ, Kaloriti D, Rancour D, Hussey P, et al. The Microtubule Plus-End Tracking Proteins SPR1 and EB1b Interact to Maintain Polar Cell Elongation and Directional Organ Growth in Arabidopsis. Plant Cell. 2014;26:4409-25.

55. Komis G, Mistrik M, Samajova O, Doskocilova A, Ovecka M, Illes P, et al. Dynamics and Organization of Cortical Microtubules as Revealed by Superresolution Structured Illumination Microscopy. Plant Physiol. 2014;165:129-48.

56. Mathur J, Mathur N, Kernebeck B, Srinivas BP, Hulskamp M. A novel localization pattern for an EB1-like protein links microtubule dynamics to endomembrane organization. Curr Biol. 2003;13:1991-7.

57. Nakamura M, Hashimoto T. A mutation in the Arabidopsis -tubulincontaining complex causes helical growth and abnormal microtubule branching. J Cell Sci. 2009;122:2208-17.
58. Nakaoka Y, Kimura A, Tani T, Goshima G. Cytoplasmic Nucleation and Atypical Branching Nucleation Generate Endoplasmic Microtubules in Physcomitrella patens. Plant Cell Online. 2015;27:1-16.

59. Wang X, Zhang J, Yuan M, Ehrhardt DW, Wang Z, Mao T. Arabidopsis MICROTUBULE DESTABILIZING PROTEIN40 Is Involved in Brassinosteroid Regulation of Hypocotyl Elongation. Plant Cell. 2012;24:4012-25.

60. Zhang Q, Fishel E, Bertroche T, Dixit R. Microtubule Severing at Crossover Sites by Katanin Generates Ordered Cortical Microtubule Arrays in Arabidopsis. Curr Biol. 2013;23:2191-5.

61. Sousa A, Reis R, Sampaio P, Sunkel CE. The Drosophila CLASP homologue, Mast/Orbit regulates the dynamic behaviour of interphase microtubules by promoting the pause state. Cell Motil Cytoskeleton. 2007;64:605-20.

62. Al-Bassam J, Kim H, Brouhard G, van Oijen A, Harrison SC, Chang F. CLASP promotes microtubule rescue by recruiting tubulin dimers to the microtubule. Dev Cell. 2010;19:245-58.

63. Maiato H, Fairley EA, Rieder CL, Swedlow JR, Sunkel CE, Earnshaw WC. Human CLASP1 is an outer kinetochore component that regulates spindle microtubule dynamics. Cell. 2003;113:891-904.

64. Bratman SV, Chang F. Stabilization of overlapping microtubules by fission yeast CLASP. Dev Cell. 2007;13:812-27.

65. Komarova YA, Vorobjev IA, Borisy GG. Life cycle of MTs: persistent growth in the cell interior, asymmetric transition frequencies and effects of the cell boundary. J Cell Sci. 2002;115(Pt 17):3527-39.

66. Small JV, Kaverina I. Microtubules meet substrate adhesions to arrange cell polarity. Curr Opin Cell Biol. 2003;15:40-7.

67. Ueda K, Matsuyama T, Hashimoto T. Visualization of microtubules in living cells of transgenicArabidopsis thaliana. Protoplasma. 1999;206:201-6.

68. Komaki S, Abe T, Coutuer S, Inze D, Russinova E, Hashimoto T. Nuclearlocalized subtype of end-binding 1 protein regulates spindle organization in Arabidopsis. J Cell Sci. 2010;123:451-9.

69. Littlejohn GR, Mansfield JC, Christmas JT, Witterick E, Fricker MD, Grant MR, et al. An update: improvements in imaging perfluorocarbon-mounted plant leaves with implications for studies of plant pathology, physiology, development and cell biology. Front Plant Sci. 2014;5:1-8.

\section{Submit your next manuscript to BioMed Central and we will help you at every step:}

- We accept pre-submission inquiries

- Our selector tool helps you to find the most relevant journal

- We provide round the clock customer support

- Convenient online submission

- Thorough peer review

- Inclusion in PubMed and all major indexing services

- Maximum visibility for your research

Submit your manuscript at www.biomedcentral.com/submit 